\title{
Modern exploration of Galileo's new worlds
}

\author{
Torrence V. Johnson \\ Jet Propulsion Laboratory, California Institute of Technology, \\ 4800 Oak Grove Dr. Pasadena CA, USA \\ email: Torrence.V. Johnson@jpl.nasa.gov
}

\begin{abstract}
Four hundred years ago Galileo turned his telescope to the heavens and changed the way we view the cosmos forever. Among his discoveries in January of 1610 were four new 'stars', following Jupiter in the sky but changing their positions with respect to the giant planet every night. Galileo showed that these 'Medicean stars', as he named them, were moons orbiting Jupiter in the same manner that the Earth and planets revolve about the Sun in the Copernican theory of the solar system. Over the next three centuries these moons, now collectively named the Galilean satellites after their discoverer, remained tiny dots of light in astronomers' telescopes. In the latter portion of the twentieth century Galileo's new worlds became important targets of exploration by robotic spacecraft. This paper reviews the history of this exploration through the discoveries made by the Galileo mission from 1995 to 2003, setting the stage for on-going exploration in the new century.
\end{abstract}

Keywords. History and philosophy of astronomy, space vehicles, planets and satellites: individual (Callisto, Europa, Ganymede, Io, Jupiter)

\section{Introduction - The Era of the Telescope}

Modern exploration of Galileo's new worlds began in a very real sense with the publication of Sidereus Nuncius itself, which announced the discovery of four moons circling Jupiter. Galileo's entire approach is recognizably modern - develop improved instrumentation (the telescope), make new observations of greater precision than ever before achieved, and, most importantly, make the results and the description of the methods used widely available as soon as possible for comment and confirming observations. The

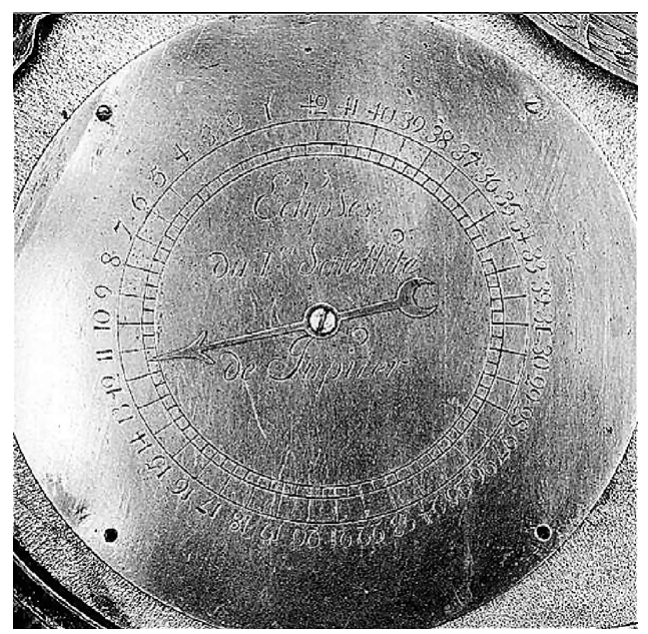

Figure 1. Io dial on planisphere in J. Paul Getty Museum collection. Note the $\sim 42.5$ marks around the circumference - approximately Io's orbital period in hours. 
Galileo scholar Albert van Helden has pointed out in his translation that Sidereus Nuncius was an entirely new kind of astronomical publication, having more in common with a modern International Astronomical Union Circular than Ptolemy's Almagest (Galilei 1610).

The telescope remained the primary tool for studying the Galilean satellites for the next three and a half centuries. As the capabilities of telescopic optics improved, so did observing techniques and the instrumentation available to augment the 'Mark-I eyeball'. Before the end of the seventeenth century, accurate timing of Galilean satellite eclipses by Danish astronomer Ole Roemer (working at the Paris Observatory) provided the means to determine the first reliable value for the speed of light, a problem that Galileo himself had been unable to solve. Thus, within a few decades of Galileo's initial observations, his 'new worlds' were providing a key that would eventually lead to the modern world of relativistic physics.

These seventeenth century observations were accurate enough that they are still used in analyzing the long term variations of the orbits of the Galilean moons. Eclipse timings for Jupiter's moons also provided a cosmic clock which enabled accurate longitudes on Earth to be determined. A testament to the pervasiveness of Galileo's moons in the culture of the Enlightenment is found in a beautiful planisphere from the mid-eighteenth century. This device, now in the decorative arts collection of the J. Paul Getty Museum, includes a specialized dial which indicates the times of eclipses for Io, the innermost of the Galilean satellites (Wilson et al. 1996).

The next centuries saw the moons studied with ever increasing sophistication as the science of astronomy developed. The photographic plate, for precision measurements of position, the spectrograph, for analyzing the color of reflected light, and Michelson's stellar interferometer, for precision diameter measurements, were all applied to studies of Jupiter's four big satellites. In the twentieth century electronic instrumentation increased the power of telescopic observations many fold. The colors of the Galilean moons were measured, near-infrared photoelectric spectra identified water ice on the surfaces of Europa and Ganymede, and accurately timed stellar occultations determined the diameters of Io and Ganymede to a few kilometers.

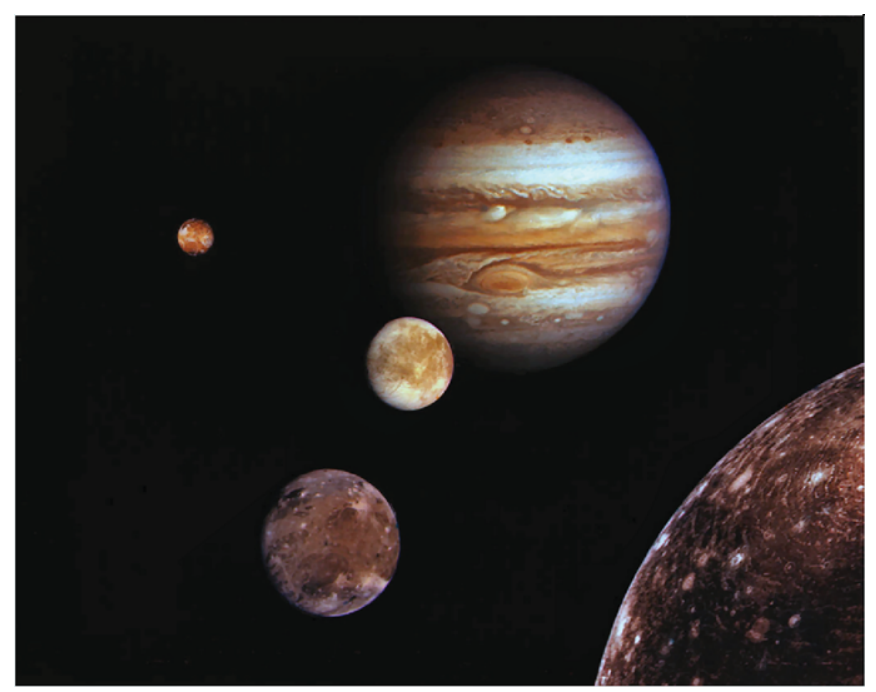

Figure 2. Voyager's 'family portrait' of the Jupiter system. Montage of images from Voyager's cameras. PhotCourtesy NASA/JPL-Caltech. 
Thus, at the beginning of the era of deep-space spacecraft exploration, in the 1970's, Galileo's new worlds, seen for so long only as points of light, were at last beginning to emerge as a reasonably well characterized, diverse set of planetary bodies. Their sizes were known, ranging from about the diameter of Earth's Moon, to somewhat larger than the planet Mercury. The available mass estimates and derived density for each satellite showed that while Io and Europa were mostly rocky objects, Ganymede and Callisto were likely an approximately even mixture of ice and rock. Io's highly reflective yellow-brown surface contrasted sharply with the less colored, icy surfaces of Europa and Ganymede and with the darker surface of Callisto. Clouds of both neutral sodium atoms and sulfur ions had been detected associated with Io. This was the sketchy but highly intriguing information which formed the basis for planning the Voyager mission's historic exploration of Jupiter's moons. The state of knowledge about the satellites at this time is reviewed in Planetary Satellites (1977).

\section{Known Worlds - The Era of Spacecraft Exploration Begins}

The Pioneer 10 and 11 spacecraft flew by Jupiter in 1973 and 1974, providing critical reconnaissance and scientific information about Jupiter's intense radiation belts, stunning views of Jupiter's clouds and glimpses of the satellites. Tracking data also improved estimates of the moons' masses significantly, confirming Io's rocky nature and Ganymede's low density and large ice content. By the time of the launch of Voyager 1 and 2 in 1977,
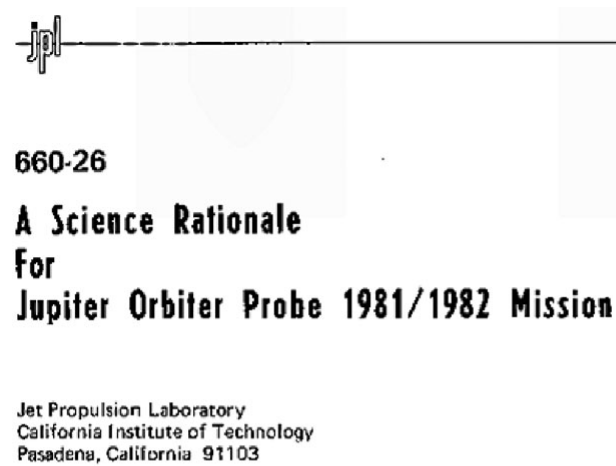

Figure 3. Cover of report recommending science objectives for the JOP, later Galileo, mission. 
the four Galilean satellites discovered in 1610 had become critical targets for investigation by the robotic explorers. The trajectories of the two spacecraft were carefully designed to allow each to have close encounters with several satellites on their way through the system. This 'celestial billiard shot' was enabled by the satellites' resonant orbits, which result in regularly recurring orbital configurations - a phenomena first explained and studied by Laplace in the eighteenth century.

Expectations were high for the first close-up views of these four planetary-scale worlds. Voyager's observations of the satellites from the multiple flybys in 1979 nevertheless exceeded even the most optimistic hopes of the mission's science teams. Images and other data from the two spacecraft revealed each satellite to have distinctly different characteristics and seemly different geologic histories. They had become, in the course of a few months, terrae cognitae, new worlds which could be mapped and studied in detail.

Io was found to be a young volcanic world with multiple active eruptions in progress during the Voyagers' visits. This activity confirmed the tidal heat source predicted by Stan Peale and his colleagues just months before the Voyager encounters, and the sulfurous emissions detected by spectrometers provided clues to the moon's bright, highly colored surface. In contrast, Callisto's dark surface was saturated with impact craters of all sizes, indicative of an ancient cold crust dating back to the near the formation of the solar system. Ganymede, the largest of the moons, appeared like a more youthful version of Callisto, with large regions of darker, heavily cratered terrain cut by bright bands of more recent origin. Europa, slightly smaller than Earth's Moon, was perhaps the biggest surprise. In Voyager's images, it's bright relatively smooth icy surface was crisscrossed by ridges and lineaments suggestive of global fracture patterns. At the resolution of the Voyager cameras - a few kilometers for the closest flyby - very few impact craters were detectable, evidence for a geologically young surface and some form of global resurfacing process. Theoretical models showing that liquid water oceans might exist under the icy crusts of the satellites provided one possible explanation for these findings. Voyager's results for the Galilean satellites are reviewed in Satellites of Jupiter (1982) and Satellites (1986).

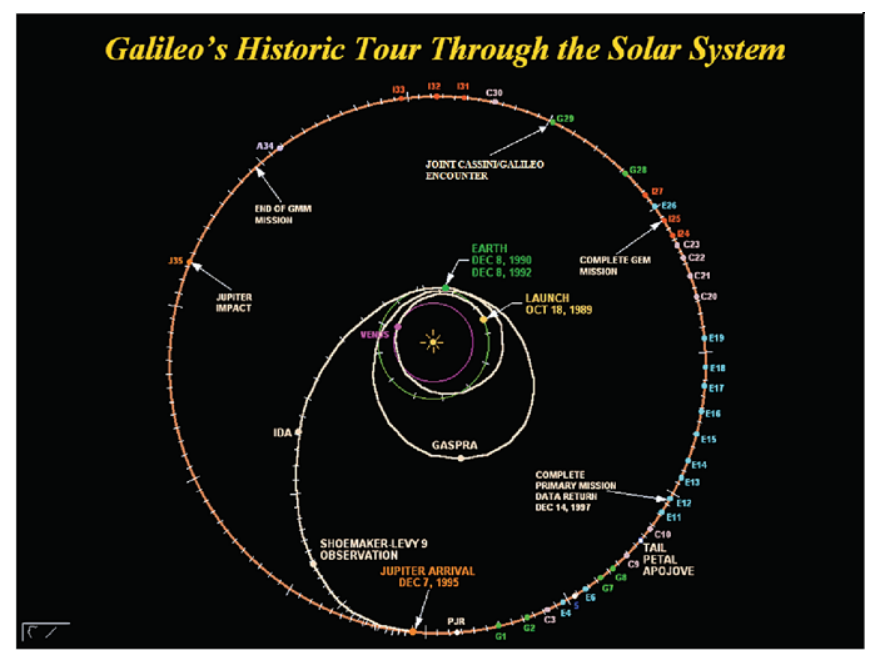

Figure 4. Galileo's mission overview. View of trajectory from N. ecliptic pole. After orbit insertion at Jupiter, each of Galileo's encounters with a satellite is marked with a dot at Jupiter's location for that date, showing the satellite encounters $(\mathrm{I}=\mathrm{Io}, \mathrm{E}=\mathrm{Europa}, \mathrm{G}=$ Ganymede, $\mathrm{C}=$ Callisto) and the orbit number. 


\section{Galileo's Namesake Explores Galileo's Worlds}

Even while preparations were being made for the launch of the Voyager spacecraft, a return to Jupiter was being planned, this time with a spacecraft that would remain in the Jupiter system in orbit around the giant planet. Although Voyager was expected to accomplish much, several scientific committees recommended that this reconnaissance needed to be followed by an orbital mission capable of studying the whole system in detail over several years. This mission, initially dubbed the JOP (Jupiter Orbiter Probe) mission was begun by NASA in late 1977, with major international contributions from Germany. Given its goal of studying the 'mini-solar system' of Jupiter, its moons and radiation environment, it was fittingly renamed Galileo to honor the discoverer of the planet's satellite system.

The Galileo mission was delayed from its initial target launch date of January 1982, first by delays in the development of the space shuttle system and the necessary upper stage rockets needed to reach Jupiter, and then by the tragic loss of the Challenger and its crew in 1986. It was eventually launched on the space shuttle Atlantis in October of 1989, and used a gravity assist trajectory involving a Venus flyby and two passes by the Earth to acquire the extra energy needed for the trip to Jupiter. On December 7, 1995 the Galileo spacecraft arrived at Jupiter. In the span of few hours, Galileo conducted its first satellite flyby of Io, recorded data from deep in Jupiter's atmosphere as the entry Probe descended on its parachute, and fired its main engines to put the craft into orbit about Jupiter, becoming the first artificial satellite of a giant planet.

Once in orbit Galileo began what became a seven year long tour of Jupiter's system, making a close flyby of one of the Galilean satellites during each orbit. Each satellite encounter was design to set up the conditions for the next encounter, changing the spacecraft's trajectory using the gravity assist technique. This permitted multiple opportunities for scientific observations of the satellites from ranges up to a thousand times closer than Voyager had achieved. The first of these encounters after achieving orbit was with Ganymede, with the spacecraft skimming low over a dark, relatively older area known as Galileo Regio, appropriately enough.

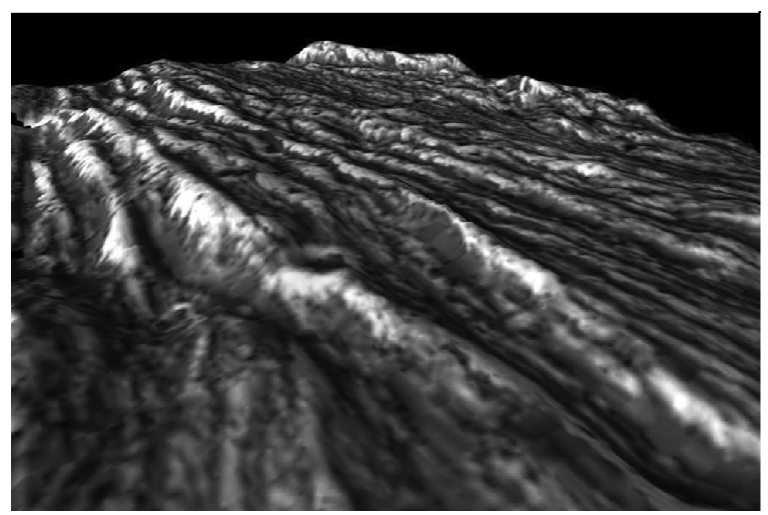

Figure 5. Galileo's view of Uruk Sulcus on Ganymede showing highly faulted surface in this shape from shading 3D rendering (approximately 3:1 vertical exaggeration. Courtesy NASA/JPL-Caltech. 
High resolution pictures of the bright, younger appearing Uruk Sulcus on the southern border of Galileo Regio resolved one of the outstanding questions from the Voyager results. Based on Voyager's lower resolution images, it had been suggested that the sulchi were possibly smooth cryovolcanic flows of water and ice filling in faulted troughs. Galileo's images showed that, to the contrary, the entire surface of Uruk Sulcus was disrupted by many small, parallel faults, destroying older crust and craters through tectonic resurfacing.

Galileo's first close encounter with Ganymede also produced the first major discovery of the mission. Measurements by the magnetometer and plasma wave experiments showed that Ganymede was a 'moon with magnetism'. During the encounter, the magnetometer recorded strong distortions in the surrounding Jovian magnetic field while the plasma wave data showed a variety of signals indicative of high energy electrons interacting with a magnetosphere. Initial calculations suggested that Ganymede possesses its own, internally generated magnetic dipole field, strong enough to stand off the local Jovian field and create a 'magnetic bubble' around the satellite - a 'magnetosphere within a magnetosphere'. Subsequent encounters with Ganymede confirmed this model and Ganymede is now believed to be the only known moon with an active dynamo generated magnetic field. Gravity data support this interpretation, showing that Ganymede is differentiated, and probably has a core of iron or iron sulfide. This finding raises interesting and still unanswered questions about how Ganymede can maintain a hot, electrically conducting, convecting liquid core this long after its formation.

Volcanic Io was not ignored by the mission planners. Although close encounters with Io early in the mission were ruled out by concerns over the radiation environment that close to Jupiter, the spacecraft performed well in the Jupiter environment, and a series of close encounters were planned during the extended mission phase. Combined with more distant observations made during the primary mission, images and thermal data showed that Io's striking eruptions were continuing, apparently unabated since the Voyager encounters in 1979 .

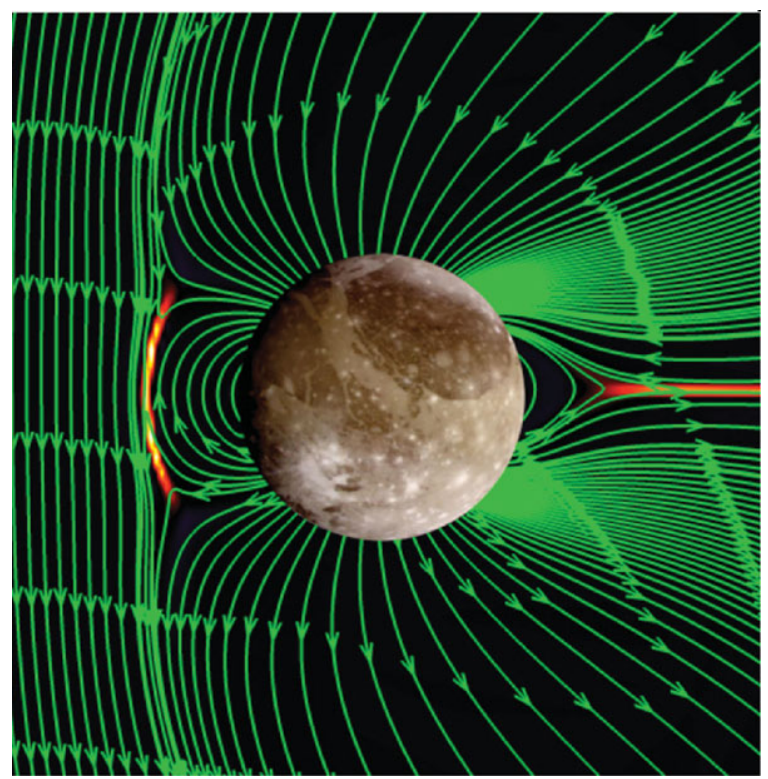

Figure 6. Ganymede's magnetosphere based on models by M. Kivelson et al. (Kivelson et al. 1997). 
Many of the active volcanic sites discovered by Voyager were still erupting during the course of the Galileo mission and multiple new eruptions were also seen. One of these new eruptions, at a location in the northern region known as Tvashtar Catena, was observed to be still active some years after the end of the Galileo mission when the New Horizons spacecraft, on its way to Pluto, turned its cameras on Io during its flyby.

The composition of the volcanic flows on Io was an unresolved issue following the Voyager observations. The relatively low temperatures of some volcanic areas measured by Voyager's infrared experiment and the ubiquitous sulfur-colored deposits on the surface led to the suggestion that Io's volcanoes might be driven by liquid sulfur rather than the higher temperature molten silicates common in terrestrial basaltic eruptions. Telescopic infrared observations from the Earth had detected higher temperatures than seen by Voyager, showing that least some of Io's activity involved silicates, but the relative importance of the two styles of volcanism on the satellites remained uncertain. Galileo instrumentation included improved near-infrared capabilities for the CCD camera and the near-infrared mapping spectrometer. These near-infrared observations confirmed that most of Io's volcanic activity involves mafic or ultramafic silicate lavas, although there may be sulfur related flows in some areas.

Given theoretical models suggesting the possibility of a liquid water ocean beneath its icy crust, enigmatic Europa was a high priority target for Galileo observations. Images from Galileo confirmed the geologic youthfulness reported by Voyager. Even with much higher resolution images available, Galileo mapping shows only a handful of large craters ( 10-20 km diameter) and many of the sprinkling of smaller craters seen at the highest resolution may be secondaries from the larger impacts. Estimates of the surface age from these results range from $\sim 50-100 \mathrm{Myr}$, implying major resurfacing of essentially the entire surface in the recent geological past. In addition, Europa's surface seen at high resolution by Galileo resembles a frozen arctic landscape, with areas of disrupted 'chaos' highly reminiscent of broken sea ice in Earth's arctic regions. Gravity data suggests that the low-density outer layer of the moon is approximately $100 \mathrm{~km}$ thick.

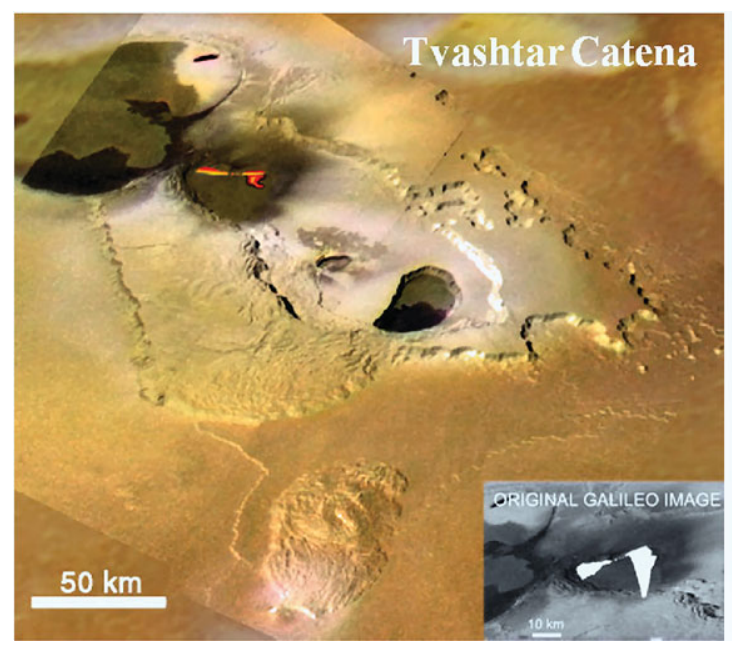

Figure 7. High temperature silicate eruption in Tvashtar Catena on Io. The infrared emission from the eruption site (marked in red) was so intense that the original CCD image was saturated in places (white "bleeding" in lower right inset). Courtesy NASA/JPL-Caltech. 
This is consistent with models having a global liquid water layer under the surface, but the gravity measurements alone cannot determine how much of this layer consists of ice versus liquid water.

As with Ganymede, some of the most important information about the satellite's interior came from magnetic field measurements. In addition to detecting the strong intrinsic field of Ganymede, the magnetometer experiment made sensitive recordings of the effects of all of the satellites on the surrounding Jovian field during each flyby. For the three icy satellites these data showed that the field perturbations were consistent with a magnetic induction response to the changing Jovian field. Since Jupiter's dipole field is tilted about ten degrees from the planet's spin axis, each satellite experiences a varying field with a time period determined by Jupiter's rotation period and the satellite's orbital period. The perturbations in each flyby (after accounting for the intrinsic field in Ganymede's case) matched the predicted induction signature expected for an electrically conducting sphere the size of the satellite. The moons' icy surface materials and their tenuous ionospheres are calculated to have far too low a conductivity to account for the observed effect. However, liquid water oceans with dissolved salt, as in Earth's oceans, could easily provide the required electrical properties. Thus Europa, Ganymede, and Callisto may all have global liquid water layers, with the major difference that Europa's ocean would be in contact with its rocky mantle, while Ganymede's and Callisto's are predicted to be 'perched' oceans, with the water layer trapped between cold low density ice above and warm high density ice phases below.

Galileo's results are reviewed in Jupiter: The Planet, Satellites and Magnetosphere (2004).

\section{Future Exploration}

The results from the Galileo mission have laid the groundwork for future exploration of this complex system of moons. NASA and ESA have done studies of a joint mission to return to Jupiter, continue the exploration of the Jovian system and place spacecraft in orbit around both Europa and Ganymede to study their surfaces and interiors in greater detail. Four hundred years after their discovery, Galileo's worlds are still playing a central role in the continuing exploration of the solar system.

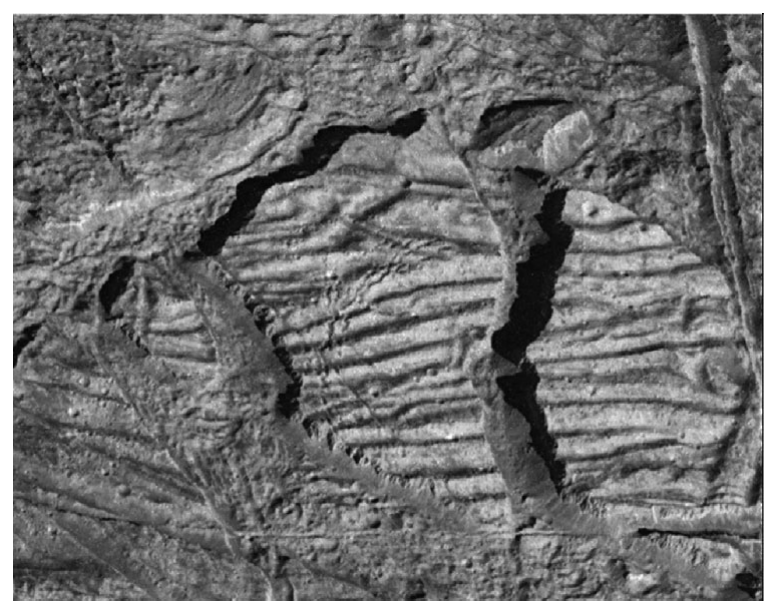

Figure 8. Ice rafts in Europa's Conamara Chaos region. Blocks are about $5 \mathrm{~km}$ across. Courtesy NASA/JPL-Caltech. 


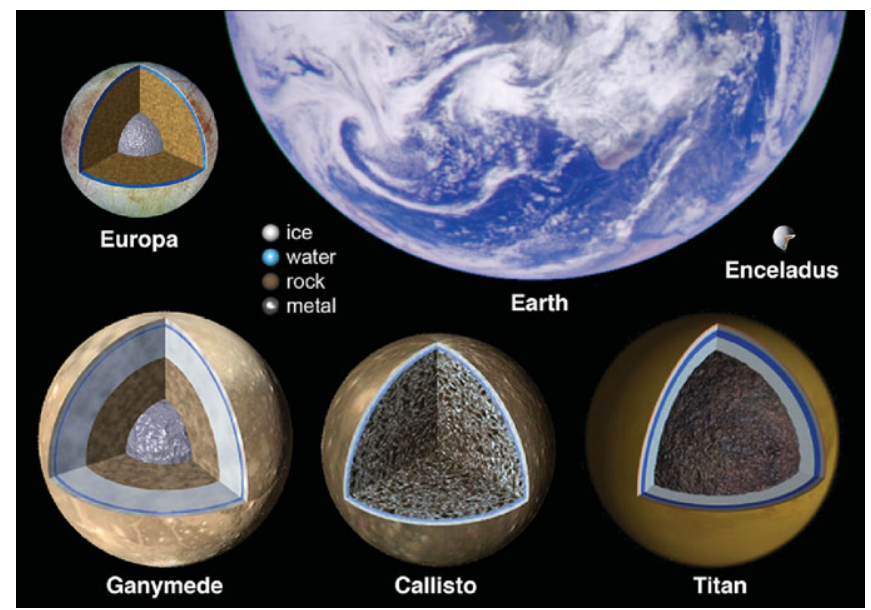

Figure 9. Planetary objects which may have oceans, to scale with Earth. Interior structures based on Galileo data and models (Galilean satellites) and Cassini/Huygens results (Titan and Enceladus). Art courtesy of Robert Pappalardo, JPL.

Acknowledgements This work was carried out at the Jet Propulsion Laboratory, California Institute of Technology under a contract from NASA.

Some of the materials used in this work were prepared for the author's presentation, The Galileo Mission: Exploring the Jovian System, at the Keck Institute for Space Studies Symposium "Challenging the Paradigm: The Legacy of Galileo" held at Caltech, November 19, 2009. http://www.kiss.caltech.edu/symposia/galileo2009/index.html

\section{References}

Burns, J. A. 1977, Planetary Satellites, Tucson: University of Arizona Press Morrision, D. 1982, Satellites of Jupiter, Tucson: University of Arizona Press Burns, J. A. \& Matthews, M. S. 1986, Satellites, Tucson: University of Arizona Press

Bagenal, F., Dowling, T., \& McKinnon, W. 2004, Jupiter: The Planet, Satellites and Magnetosphere, New York: Cambridge University Press

Galileo, G. 1610, Sidereus Nuncius, Chicago: The University of Chicago Press

Kivelson, M. G., Khurana, K. K., Coroniti, F. V., Joy, S., Russell, C. T., Walker, R. J., Warnecke, J., Bennett, L., \& Polanskey, C. 1997, Geophys. Res. Lett., 24, 2155

Wilson, G., Cohen, D. H., Ronfort, J. N., Augarde, J.-D., \& Friess, P. 1996, European Clocks in the J. Paul Getty Museum, Los Angeles: The J. Paul Getty Museum 